\title{
Stimulus generalization in monkeys following discrimination training with gray stimuli*
}

\author{
STEVEN F. FLAGG \\ Washington State University, Pullman, Washington 99163 \\ DOUGLAS L. MEDIN \\ Rockefeller University, New York, New York 10021 \\ and \\ ROGER T. DAVIS $\dagger$ \\ Washington State University, Pullman, Washington 99163
}

\begin{abstract}
Eight rhesus monkeys were trained on a successive discrimination problem with light gray and dark gray stimuli. Following training, generalization tests were given with variable stimuli ranging from white to black. Responses and response latencies were recorded. Responses were faster to the training stimulus values than to middle grays or to the extreme values on the lightness continuum. The theoretical implications of this as compared to other possible findings are developed.
\end{abstract}

How does a monkey decide which response to make in a choice situation? This question may be asked in the context of stimulus generalization following discrimination training. Some previous evidence suggested that monkeys use only a minimal amount of information in generating choices, but no evidence was provided concerning alternative descriptions of just what this information might be. Medin, Borkhuis, \& Davis (1970) trained monkeys in a black-white successive discrimination paradigm followed by transfer tests employing middle grays. Monkeys responded in a graded manner (see also Davis, Masters, \& Tjomsland, 1965): The closer a gray was to black, the more likely Ss were to judge it black. The latency function was curvilinearly related to the shade of gray, with the longest latencies appearing for the middle grays. These data as well as response patterns of individual Ss supported a "vicarious trial and error" (VTE) random walk model (adapted from Bower, 1959) which implies that Ss look to one alternative, make a yes-no decision, and so on until a response is made. This model implies that information is not used in a cumulative fashion, since the decision at each stage was assumed to be independent of any previous decision or information. In contrast, the predictions of a recruitment model (LaBerge, 1962), which assumes information is accumulated prior to a choice, were not supported by the data.

The present study was designed to provide more details concerning the choice process in a situation that seems to be more natural for models described in terms

\footnotetext{
*This research was supported by United States Public Health Service Grant MH20098-01 to Douglas L. Medin and HD05902-03 to Roger T. Davis.

$\nmid$ Requests for reprints should be sent to Roger T. Davis, Department of Psychology, Washington State University, Pullman, Washington 99163.
}

of accumulating information. Monkeys were trained to respond to the left stimulus when presented with two light-gray stimuli and to the right when presented with two dark-gray stimuli. After training to a strict criterion of learning, tests for stimulus generalization to eight different transfer stimuli ranging from white to black were given. Responses and response latencies were recorded for each of the eight transfer stimuli.

According to the recruitment theory (LaBerge, 1962), we can view different stimuli along the lightness continuum as being made up of different proportions of white and black elements, depending on the lightness of the stimuli. When presented with one of the training stimulus values, the probability of sampling one type of element or the other depends on the relative proportions of the two kinds of elements in the stimulus. Elements are randomly sampled one at a time, and the information from these samples is accumulated until a decision is made. If a certain fixed number $K$ of white elements are sampled before $\mathrm{K}$ black elements, the appropriate response for the light-gray stimulus is made. For a simple black-white discrimination, $\mathrm{K}$ could be as small as one since only black elements could be sampled from the black stimulus and only white elements from the white stimulus. However, in order to achieve the near-perfect performance on the training problem required by the stringent learning criterion of the present study, $\mathrm{K}$ must be quite large to insure that the probability of sampling $K$ black elements from the dark-gray training stimulus before $\mathrm{K}$ white elements (and the opposite for the light-gray training stimulus) is close to one. Since $\mathrm{K}$ has to be large enough for nearly perfect performance on the training problem, the Ss should show nearly perfect performance during generalization testing on stimuli having the same Munsell values as used in training and any stimulus with more extreme values. 
However, since the intermediate transfer stimulus values require higher Ks for perfect performance, the monkeys should show somewhat poorer performance on them. The result will be a generalization gradient for the choice data that is " $S$ " shaped, with the tails of the " $S$ " being horizontal. If training were not given to a strict criterion, Ss might make fewer "errors" on the extreme stimuli than on the training stimuli, since $K$ need not be so large for the extreme values to insure perfect responding.

If we assume that it takes one unit of time to sample each element, LaBerge's theory would predict an inverted $U$ shape for the stimulus generalization gradient of response latencies. This follows from the fact that, in order to sample $\mathrm{K}$ black elements, more total elements must be sampled from a dark-gray stimulus than from a black stimulus because some of the elements sampled from the gray stimulus will be white. The largest number of elements will be sampled from the middle grays, and the smallest number from the extreme values of black and white before a decision can be made. This will lead to long response latencies for the middle grays and decreasing latencies toward the extreme values. In summary, theories assuming that an accumulation of information occurs prior to a choice lead to the prediction of an inverted U-shaped gradient for response latencies on the generalization tests.

Some models assuming that monkeys do not use information in a cumulative manner would also predict an inverted U-shaped latency gradient. Any theory that states that Ss discriminate by responding to relative cues would make this prediction if one assumes that greater differences or contrast in lightness of cues is associated with easier and faster decisions. Likewise, the signal recognition theory employed by Heinemann, Avin, Sullivan, and Chase (1969) yields an inverted U-shaped latency function, if animals responded faster, the greater the distance between a test stimulus and the cutoff or criterion point for decisions.

There are other theories assuming information is not accumulated prior to a choice that predict W-shaped generalization gradients for response latencies. The simplest theory of this type is a straightforward recognition theory. According to this theory, the monkey learns that for one specific training value a left response is rewarded and for the other a right response is rewarded. When presented with another stimulus during generalization testing, the monkey decides if it looks like the specific dark-gray training stimulus or like the light-gray training stimulus. If we assume that the farther the transfer stimulus is from the training stimulus the harder it is to decide which training stimulus it looks like and the longer it takes to decide, then the shortest response latencies during generalization testing should be to the training stimulus values. The stimuli at the extremes of the continuum and those between the training values should have longer response latencies, giving rise to a W-shaped generalization gradient of response latencies.
During generalization testing, the monkeys should be less likely to judge stimulus values correctly that fall midway between the two training stimulus values. The result will be an S-shaped curve for the choice generalization gradient. The exact shape depends on what the monkey does when presented with the extreme stimulus values of black and white. Suppose, for example, that the monkey is presented with a black stimulus. Since this stimulus is different from the dark-gray training stimulus, the monkey may decide that it does not look like the dark-gray stimulus but, when faced with the alternative of calling it light gray, it decides the stimulus must be dark gray after all. If this is the case, the generalization curve for the choice data will be $S$ shaped with horizontal tails. However, it may also be reasonable to assume that if the monkey is faced with a black stimulus and decides it is not like the dark-gray stimulus, it may decide that it does not look like the light-gray stimulus and simply respond at random. This assumption would result in the tails of the $S$ curve turning down and up.

Theories of discrimination learning based on gradients of excitation and inhibition, such as Spence's (1936), can make similar predictions. Since the present experiment involved a successive discrimination, Spence's theory is not entirely applicable because the original theory predicted that a successive discrimination would be insoluble. However, using the logic of generating excitation and inhibition gradients, the theory can predict either W-shaped or U-shaped latency functions but not inverted U-shaped functions. Although the present study is more or less tangential to the phenomena of transposition, in the successive paradigm used here transposition or peak shift cannot be predicted on the basis of excitation and inhibition gradients. The only values that would yield faster responding to stimulus values away from the training stimulus predict that responding should be still faster to the middle training stimuli. There is very little evidence available concerning transposition in the successive discrimination paradigm used in the present experiment (see Reese, 1968, for a review).

The present experiment recorded both responses and response latencies during generalization testing in order to test the predictions of the various theories.

\section{METHOD}

\section{Subjects}

The Ss were five old and three middle-aged rhesus monkeys. The middle-aged animals ( 15 to 17 years) were progeny of the older (approximately 20 to 21 years old) jungle born animals. All the animals had received nearly identical training for the last 12 years on numerous laboratory experiments concerned with learning and perception. 


\section{Apparatus}

A Wisconsin General Test Apparatus (WGTA) was used for testing the monkeys. The formboard was covered with medium-gray Munsell paper (Value 5.5) and slanted forward $50 \mathrm{deg}$ to diminish surface reflectance. The two foodwells were $8 \mathrm{in}$. apart, center to center. Illumination was provided by two 20-W fluorescent lights, one over the formboard and one over the animal's restraining cage. The reflectance reading at the foodwells was $9.36 \mathrm{fc}$, as measured by a Macbeth illuminometer.

Stimuli were gray matte-finished Munsell papers glued to 2-in. pressed board squares. The Munsell Values employed were 4 and 7 for the training stimuli and 2 (black), 3, 4, 5, 6, 7, 8, and 9 (white) for the transfer tests.

Response latencies were measured by a timer that started when the opaque door of the WGTA was raised to initiate a trial and stopped when the monkey broke a photocell beam by displacing one of the stimuli.

\section{Procedure}

\section{(1) Training}

All the monkeys were trained on a successive discrimination problem with stimuli covered with Munsell paper of Value 4 or 7. For half the monkeys, if both foodwells were covered with Value 4 stimuli, a response to the left-hand stimulus was correct; if the foodwells were covered with Value 7 stimuli, the appropriate response was to the right-hand stimulus. Half of the monkeys were trained with the opposite signs and had to respond right for identical stimuli of Value 4 and left for Value 7. A noncorrection procedure was used and correct responses were rewarded with a raisin.

Each monkey received 50 trials per day until the criterion of at least 23 correct responses to each stimulus value for 2 successive days was met. The order of appearance of the two stimulus values was randomized daily, with the constraint that each occur 25 times per day. The intertrial interval was approximately $10 \mathrm{sec}$.

\section{(2) Transfer Tests}

The transfer tests, which began the day after a monkey met the learning criterion, consisted of 16 three-trial problems per day for 6 days. The first two trials of each problem were practice trials and consisted of one trial with each of the two training stimulus values. The training stimulus value given on each practice trial was randomly determined, and the reward contingencies remained the same as in training. The third trial of each problem was a nondifferentially rewarded transfer test trial,

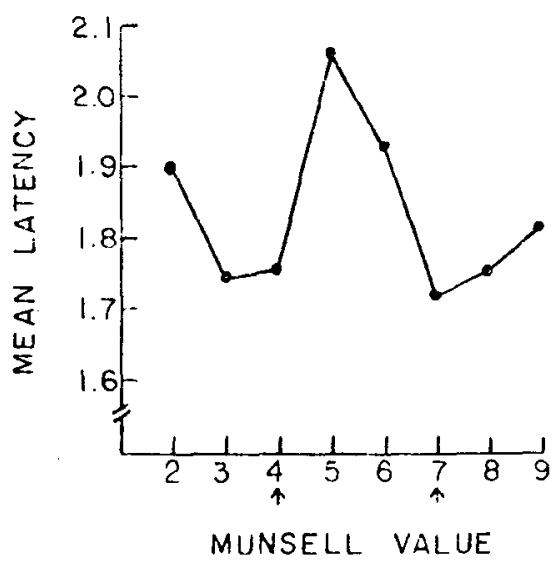

Fig. 1. Generalization gradient for the response latency data. Arrows indicate Munsell values used for training.

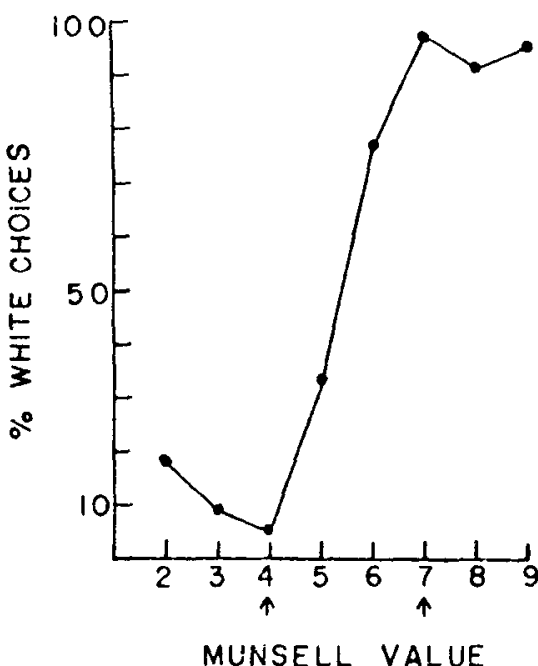

Fig. 2. Generalization gradient for the choice data. Arrows indicate Munsell values used for training.

and the stimulus value used was randomly chosen from the transfer test Values 2 through 9, with the constraint that each value appear in two problems per day. One additional condition was that, when Munsell Values 4 or 7 appeared on the critical third trial of a problem, the value used on the second trial of that problem was always the other training stimulus value. Responses and response latencies were recorded by the $\mathrm{E}$ throughout the experiment.

\section{RESULTS}

Mean response latencies from the generalization tests are plotted in Fig. 1. The curve in Fig. 1 definitely appears to be $\mathrm{W}$ shaped, rather than in the form of an inverted U. In Fig. 2, percent of choices to the side appropriate for the light-gray training stimulus is shown as a function of transfer stimuli. The curve appears to be $S$ shaped, with the upper and lower tails curving down and up, respectively. The precise shape of these curves will be more carefully analyzed in the following formal analysis.

For purposes of analysis, the 6 days of transfer tests were broken into two blocks of 3 days each in order to assess the effect of practice on the generalization tests. The resulting two within-Ss factors were transfer stimuli and practice. An analysis of variance performed on the choice data showed that the effect of transfer stimuli was highly significant $(\mathrm{F}=84.38$, $\mathrm{df}=7 / 49, \mathrm{p}<.01)$. Neither the effect of practice $(F<1)$ nor the Practice by Transfer Stimuli interaction $(F<1)$ were significant. The response latency data gave a similar picture, with the effect of transfer stimuli again being significant $(\mathrm{F}=2.88, \mathrm{df}=7 / 49, \mathrm{p}<.05)$ and the Transfer Stimuli by Practice interaction being nonsignificant $(F=1.25$, $\mathrm{df}=7 / 49, \mathrm{p}<.10)$. However, the practice effect, which was not significant for the choice data, did show a significant effect on the latency data $(F=6.18, \mathrm{df}=1 / 7$, $\mathrm{p}<.05)$. The significant practice effect indicates that the monkeys' response latencies changed over the 6 days of transfer tests. However, this change was in the form 
of shorter response latencies to all transfer stimuli and. indeed, the nonsignificant Practice by Transfer Stimuli interaction indicates that the change was not significantly different for different stimuli.

A trend analysis (Winer. 1962) was used to determine if the apparent $W$ shape of the curve in Fig. I was reliable. The only component of the analysis that showed a significant $F$ value was the quartic $(F=9.50$. $\mathrm{df}=1 / 49, \mathrm{p}<.01$ ), which is consistent with a W-shaped response latency generalization gradient.

The application of trend analysis to the generalization gradient for the choice data (Fig. 2) is somewhat more complicated. If one used a trend analysis on percent white choices, one would expect a significant cubic component that would not differentiate the various theoretical predictions. Therefore, for purposes of the trend analysis, the choice data (number of times each stimulus was responded to as if it were light gray) were converted to number of errors for each transfer stimulus. An error was somewhat arbitrarily defined as responding in the direction appropriate for training stimulus Value 4 when presented with transfer stimuli of Values 6 through 9. Likewise, it was considered an error when the monkeys responded in the appropriate direction for training Value 7 when presented with transfer Values 2 through 5. A trend analysis showed that the only significant component was the quartic $(F=10.00$, $\mathrm{df}=1 / 49, \mathrm{p}<.01)$, indicating that the error data generalization gradient is compatible with a W-shaped curve.

\section{DISCUSSION}

The results of this study are relatively clear in showing a W-shaped response latency gradient and support the conclusions from the Medin et al (1970) experiment that monkeys do not use information in a cumulative manner in this paradigm. The data are consistent with a VTE model assuming that a decision is independent of any previous decisions or information.

While it seems that one should favor the models predicting the W-shaped latency gradient observed in this experiment, the generality of these conclusions is somewhat limited by a lack of unanimity of results from previous investigations. Heinemann, Avin, Sullivan, and Chase (1969) and Heinemann and Chase (1970) have obtained choice curves favoring a signal detection theory analysis using both successive discriminations and the single-response "go/no-go" paradigm with pigeons as Ss. On the other hand, results like those of the present study have been obtained by Ernst, Engberg, and Thomas (1971) with pigeons, by Thomas and Setzer (1972) with rats and guinea pigs, and by Moody, Stebbins, and Iglauer (1971) using monkeys.
Athough the present study used a successive discrimination paradigm, the experimental situation offered an excellent opportunity for relational responding because the background food-tray color was midway between the two training stimuli in lightness. The monkey could have learned during training to respond in one direction for stimuli darker than the background and in the other direction for stimuli lighter than the background. Perhaps if the training stimuli were less identifiable, one would observe more relative responding.

This is probably not the best place to speculate on the various possibilities for when one rather than the other result ought to occur, especially since Thomas and Setzer (1972) have already brought out many of these methodological differences. Still the picture is unclear and to attempt any broad generalizations may only serve to cloud the issues. It remains for further work to sort out or isolate conditions (procedures, stimuli, species) under which one form of responding or another is observed.

\section{REFERENCES}

Bower, G. H. Choice-point behavior. In R. R. Bush and W. K. Estes (Eds.), Studies in mathematical learning theory. Stanford, Calif: Stanford University Press, 1959.

Davis, R. T., Masters, H. G., \& Tjomsland, J. Perception by monkeys: I. Psychophysical judgments of brightness by human and subhuman Ss. Perceptual \& Motor Skills, 1965, 20,637-745.

Ernst, A. J., Engberg, L., \& Thomas, D. R. On the form of stimulus generalization curves for visual intensity. Journal of the Experimental Analysis of Behavior, 1971, 16, 177-180.

Heinemann, E. G., Avin, E., Sullivan, M. A., \& Chase, S. Analysis of stimulus generalization with a psychophysical method. Journal of Experimental Psychology, 1969, 80, 21 5-224.

Heinemann, E. G., \& Chase, S. On the form of stimulus generalization curves for auditory intensity. Journal of Experimental Psychology, 1970, 84, 483-486.

LaBerge, D. A recruitment theory of simple behavior, Psychometrika, 1962, 27, 375-396.

Medin, D. L., Borkhuis, M. L., \& Davis, R. T. Response latency and brightness judgments by monkeys. Journal of Experimental Psychology, 1970, 83, 480-485.

Moody, P. B., Stebbins, W. C., \& Iglauer, C. Auditory generalization gradients for response latency in the monkey. Journal of the Experimental Analysis of Behavior, 1971, 16, $105-111$.

Reese, H. W. The perception of stimulus relations. New York: Academic Press, 1968.

Spence, $K$. W. The nature of discrimination learning in animals. Psychological Review, 1936, 43, 427-449.

Thomas, D. R., \& Setzer, J. Stimulus generalization gradients for auditory intensity in rats and guinea pigs. Psychonomic Science, 1972, 28, 22-24.

Winer, B. J. Statistical principles in experimental design. New York: McGraw-Hill, 1962.

(Received for publication April 18, 1973; revision accepted September 17, 1973.) 